\title{
L'INFINITISME PANTHÉISTE DE JOHN TOLAND ET SES RELATIONS AVEC LA PENSÉE DE GIORDANO BRUNO
}

\author{
Jean SEIDENGART
}

\begin{abstract}
Résumé : Il s'agit de repartir de la découverte et de l'acquisition, par John Toland, des principaux écrits de Giordano Bruno afin de déterminer l'impact de la philosophie nolaine sur la pensée du philosophe irlandais. Plus précisément, nous analyserons 1'interprétation tolandienne de l'œuvre de Bruno qui figure dans le mémoire qu'il adressa à ce sujet à son ami le baron Hohendorf. De là, nous dégagerons les grandes lignes de l'infinitisme panthéiste qui figurent dans les écrits ultérieurs de Toland pour tenter d'identifier, d'une part, ses emprunts au philosophe italien et, de l'autre, sa façon d'intégrer ou d'incorporer à sa propre pensée ces divers philosophèmes bruniens, en versant ainsi du vin nouveau dans de vieilles outres.
\end{abstract}

Mots clés : cosmologie, infini, panthéisme, nature, matière, immanence.

SUMmaRY: Here, the point is to go back to the discovery and the purchase by John Toland of Giordano Bruno's most important writings in order to assess the influence of Brunian philosophy on the Irish thinker. To be more precise, this article wants to analyse Toland's interpretation of Bruno's work, which appears in the paper he sent about it to his friend, the lord of Hohendorf. Then will be drawn the broad outlines of the pantheistic infinitism that Toland develops in his other works, in order to determine, on the one hand, what has been borrowed from Bruno, and, on the other hand, his peculiar way of integrating these Brunian tenets into his own philosophy.

Keywords : cosmology, infinity, pantheism, nature, matter, immanence. 
Zusammenfassung: Ausgehend von der Tatsache, daß John Toland die Hauptwerke Giordano Brunos für sich entdeckt und erworben hat, wird in diesem Artikel untersucht, welchen Einfluß die Philosophie Giordano Brunos auf den irischen Denker ausgeübt hat. Am Beispiel der Abhandlung über Bruno, die Toland seinem Freund, dem Freiherrn von Hohendorf, zugeeignet hat, soll gezeigt werden, wie Toland Brunos Werk interpretiert hat. Anschließend werden die Grundzüge des pantheistischen Infinitismus beschrieben, den Toland in seinen anderen Werken dargestellt hat. Dabei wird gezeigt, was er aus den Schriften Brunos entlehnt hat und wie er vorgegangen ist, um dessen Lehren in seine eigene Philosophie zu integrieren.

Stichwörter : Kosmologie, Unendliches, Pantheismus, Natur, Materie, Immanenz.

Jean SeIDENGart, né en 1949, est agrégé de philosophie, docteur d'État ès lettres et sciences humaines, professeur à l'université de Reims Champagne-Ardenne. Ses recherches portent sur l'histoire et la philosophie de la science classique, en particulier, la cosmologie, l'astronomie et la physique.

Adresse : Université de Reims Champagne-Ardenne, U.F.R. de lettres et sciences humaines, Département de philosophie, 57, rue Pierre-Taittinger, 51096 Reims Cedex. 


\section{1. - TOLAND DÉCOUVRE LES GRANDS ÉCRITS DE BRUNO EN 1698}

Grâce aux récentes recherches philologiques, historiques et bibliographiques de F. H. Heinemann ${ }^{1}$ et plus encore à celles de Giovanni Aquilecchia $^{2}$ et de Rita Sturlese ${ }^{3}$, il a été possible de faire toute la lumière sur les circonstances au cours desquelles John Toland fit l'acquisition de certains des plus grands écrits italiens de Giordano Bruno et même de la trilogie latine de Francfort. Toutefois, il ne faudrait pas en conclure pour autant que le libre penseur irlandais n'avait jamais entendu parler de Bruno ni de sa philosophie avant la date capitale de 1698. Inversement, la grande érudition dont témoignent les écrits de Toland doit nous conduire à penser que la philosophie de Bruno n'est que l'une des nombreuses composantes de sa propre philosophie, même si la référence au Nolain représente certainement l'une de celles qui exerça l'influence la plus profonde et la plus durable sur lui.

C'est, en fait, au cours d'une vente aux enchères de la bibliothèque de Francis Bernard ${ }^{4}$, qui eut lieu en 1698 , qu'un ouvrage relié réunissant plusieurs livres de Bruno fut acheté par John Toland. Cet ouvrage est l'exemplaire qui avait appartenu personnellement autrefois à la reine Élisabeth $I^{\mathrm{Te}}$, car il porte les armes d'Angleterre sur le maroquin noir de sa reliure; il réunissait en un seul volume : le Spaccio, la Cena, le De l'infinito et la Causa. Les recherches très précises de $G$. Aquilecchia ont même permis de rectifier une erreur commise par John Toland en personne à propos de la date de cette acquisition. En effet, John Toland écrivit en novembre 1709 au baron Hohendorf qu'il avait acheté le Spaccio en 1696, alors que la vente n'eut lieu qu'en 1698, donc deux ans après la publication de Christianity not Mysterious ${ }^{5}$. Revenant sur les recherches effectuées par G. Aquilec-

1. F. H. HeinemanN, « John Toland and the Age of Enlightenment », Review of English Studies, vol. XX, 78, 1944, p. 125-146.

2. Giovanni AQuLECCHIA, "Nota su John Toland traduttore di G. Bruno », English Miscellany, IX, 1958, p. 77-86.

3. Rita Sturlese, "Postille autografe di John Toland allo "Spaccio" del Bruno ", Giornale critico della filosofia italiana, LXV, 1986, p. 27-41. Cf. aussi ID., Bibliografia, censimento $e$ storia delle stampe originali di Giordano Bruno, Forence, Olschki, 1987, p. XXIV-XXVI, XXXIII et $49-50,53,56,58,63,65-66,105$.

4. Francis Bernard était un médecin de grande réputation au service du roi Jacques II d'Angleterre. Homme d'une grande érudition et polyglotte, Francis Bernard était étroitement lié aux tories et à la High Church.

5. G. AQUILECCHIA, in art. cit. supra n. 2, p. 85, publie la transcription de la note manuscrite de l'antiquaire anglais, John Bagford, qui fait état de cette vente à John Toland: «Mr. T[oland] : bought the booke out of my handes in the sale of dr Fran: Bermards Bookes in Little Brittane it was printed in at London as the Letter unquestionably proves. and if you turne to page 20 of the Cataloug part the III; you will find Number $242: 243: 244: 245$ to 
chia, Saverio Ricci a montré que John Toland devait également posséder la trilogie de Francfort ${ }^{6}$, car ce même Catalogue mentionnait ces poèmes ainsi que leur prix de vente ${ }^{7}$. Nos analyses des textes de Toland apportent une confirmation à cette hypothèse, car nous verrons plus loin quelques passages du free-thinker répétant mot pour mot certaines des formules de Bruno qui ne figurent que dans le De immenso. L'enthousiasme fidèle de Toland pour la «nolana filosofia » et son puissant réseau de relations intellectuelles à travers l'Europe constituent finalement l'un des plus importants pôles de diffusion de l'infinitisme brunien au début du Xvin' siècle.

II. - TOLAND ET L'INTERPRÉtATION PANTHÉISTE DE LA COSMOLOGIE BRUNIENNE

À la suite de ses recherches personnelles sur la vie et l'œuvre de Giordano Bruno, Toland réunit un certain nombre de documents qu'il critiqua avec une prudence et une finesse tout à fait remarquables. Parmi ceux-ci, il faut citer le petit mémoire intitulé De genere, loco et tempore mortis Jordani Bruni Nolani, qu'il adressa à son ami, le baron Hohendorf en 1709 depuis Amsterdam ${ }^{8}$. Ce texte contient la célèbre lettre que Gaspard Schopp, ce protestant converti au catholicisme et résidant à Rome au moment de la fin du procès de Bruno, envoya à son maître Conrad von Rittershausen pour lui relater les péripéties du procès d'Inquisition, les chefs d'accusation et l'exécution capitale du Nolain au Campo dei Fiori le 17 février 1600 . Cette lettre de G. Schopp contient en quelque sorte la déposition d'un témoin oculaire de l'exécution de Bruno, comme le rappelle le déiste irlandais :

be sevirall pises of Giordano: Bruno Nolana [sic] bound togeteher as the brace at the end of the Lines shows you. Nomber 244 : intitled Spattio della Bestia : Trionfante Dialogo. That is in English the dispatching of the Triumphing Beast (meaning the Revelation). tis in Italian and by the Armes all bound together it apperes to have been Queene Elezabeth owne Booke. " G. Aquilecchia s'est reporté audit Catalogue qui précise à la p. $20:$ «242 Bruno Nolano de la Causa Principio; 243 De l'Infinito, Universo e Mundo; 244 Spaccio de la Bestia Trionfante Dialogo; 245 Dialoghi la Cena de la Ceneri.»

6. C'est-à-dire le De monade, le De triplici minimo et le De immenso et innumerabilibus.

7. Saverio Ricci, La Fortuna del pensiero di G. Bruno (1600-1750), Florence, Le Lettere, 1990, IV, «Dio e natura », p. 242-243, n. $11:$ «Cf. A Catalogue Of The Library of the late Learned Dr. Francis Bernard, Fellow of the College of Physicians, and Physician to S. Bartolomew's Hospital [...] sold by Auction [...] October 4. 1698, pp. 58, nn. 55-56 per i Poermi francofortesi. [...] Sono annotati, accanto ai titoli bruniani, i prezzi pagati dall' acquirente : " $\mathfrak{f} 4$ " per i Poemi, " $£ 2.5 .0$ " per lo Spaccio e per gli altri dialoghi. »

8. Le texte de cette lettre a été publié dans l'édition posthume des œuvres de John Toland : A Collection of Several Pieces of Mr John Toland, Londres, 1726, t. 1, p. 304-328. S. RICC, in op. cit. supra n. 7, p. $268 \mathrm{sq}$., signale que le texte original manuscrit se trouve actuellement à la Nationalbibliothek de Vienne. 
« Bruniani supplicii hic habemus non auritum modo, sed magis adhuc credibilem testem, oculatum nempe, Gasparem Scioppium; qui Inquisitoribus, dum Brunus interrogaretur, aderat; quique eundem postea in Campo Florae igni devorandum, ab urbis Praefacto traditum, viderat ${ }^{9}$.

Toland ne doute pas un seul instant que Bruno ait été brûlé vif sur ordre de l'Inquisition; d'ailleurs il est même outré des réserves que Bayle avait émises sur ce point dans son Dictionnaire historique et critique en se référant à son tour à l'article «Bruno » de la Bibliotheca Neapolitana rédigé par Toppi et Nicodème ${ }^{10}$.

C'est juste après le texte de cette lettre que Toland émet des considérations critiques sur certains points de la doctrine de Bruno, tout en écartant toutes les calomnies qui défiguraient la véritable philosophie nolaine. Très habilement, Toland fait remarquer que Nicodème ainsi que Gaspard Schopp ne sont pas véritablement crédibles lorsqu'ils font état de la pensée de Bruno, puisqu'ils rapportent des propos contradictoires. Calmement, Toland dénonce au passage la technique habituelle de falsification des dépositions des personnes suspectes d'hérésie, reposant sur la mauvaise foi caractéristique des Inquisiteurs décidés à perdre les prévenus qui ne vont pas à résipiscence. Il réussit même à montrer la monstrueuse absurdité des prétendues réponses de Bruno, qui laisse apparaître clairement la machination grotesque des accusateurs :

«Or, Nicodème n'y parle pas de la mort de Brunus, mais des diverses pensées de celui-ci que les Inquisiteurs et Scioppius, qui sont de mauvaise foi < mala fide >, lui avaient attribuées; et ce qu'il y a de plus certain, c'est que ces [propos] ne sont pas tous véridiques, ni vraisemblables, parce que c'est tout à fait clair dans les écrits de celui-ci [Scioppius] et qu'il y a même une contradiction entre ses pensées. Ceci est habituel pour les Inquisiteurs et c'est une pratique fréquente que de dénigrer d'une manière odieuse au préalable ceux qu'ils désirent impatiemment perdre; de sorte qu'une fois chargés de la plupart des

9. I. TolAND, De genere, loco et tempore mortis Jordani Bruni Nolani, 1709, in op. cit. supra n. 8, t. 1, p. 311 . Le début de la lettre de Caspard ScHopp déclare, p. 305-306: «Quas ad nuperam tuam expostulatoriam epistolam rescripsi, non jam sane dubito quin tibi sint redditae; quibus me tibi, de vulgato responso meo satis purgatum confido. Ut vero nunc etiam scriberem hodierna ipsa dies me instigat. qua Jordanus Brunus propter haeresin, vivus vidensque, publice in Campo Florae, ante Theatrum Pompeii, est combustus. Existimo enim \& hoc ad extremam impressae Epistolae meae partem, qua de Haereticorum poena egi, pertinere. Si enim nunc Romae esses, ex plerisque omnibus Italis audires Lutheranum esse combustum; \& ita non mediocriter in opinione tua confirmareris, de saevitia nostra. »

10. J. Toland, op. cit. supra n. 9, p. 312, c'est nous qui traduisons : "Chez celui-ci même [s. e. Bayle], diras-tu, l'incertitude historique apparaît de plus en plus; et il ne semble pas que Bayle soit totalement dénué de preuve[s] puisqu'il écrit que Nicodème, dans les Additions à la Bibliothèque de Naples déjà rappelées, avait affirmé que tout ce qui est mentionné par Ursinus d'après la Lettre de Scioppius, n'est pas vrai, » 
vices propres à l'âme et au corps, ils sont jugés dignes non point de quelque miséricorde, mais plutôt de la haine de tous. Or, comment Brunus pourrait-il donc combattre pour défendre le salut des Démons (par exemple), comme Origène l'avait fait jadis, s'il considérait les Écritures Saintes comme des songes? Ou bien, étant donné qu'il a rejeté les Écritures, quel genre de discours pourrait-il donc tenir de son propre chef au sujet des Diables ou du salut éternel? C'est pourtant ce qu'insinuaient les Pères du Saint-Office " ${ }^{11}$.

Il faut reconnaître, bien sûr, que la confiance que Toland accordait à la cohérence de la pensée brunienne reposait sur la lecture de ses cuvres et non pas sur des témoignages plus ou moins suspects de ses contemporains. Dès lors, Toland s'efforce de montrer le bien fondé de la philosophie brunienne en reconduisant certaines expressions ou certains écrits à leur sens authentique; le ton de John Toland se fait ici nettement plus militant en faveur du Nolain. C'est ainsi, par exemple, qu'il explique à son correspondant que le terme de magie ou même celui de transmigration qui figure à maintes reprises dans les écrits de Bruno ne doit pas être pris dans un sens obscur ou mystérieux ${ }^{12}$.

Toland reproche à G. Schopp de n'avoir pas su distinguer dans les Dialogues italiens entre les propos des divers personnages et ceux qui expriment la nolana filosofia. Il faut bien reconnaître que ce n'est pas toujours chose aisée, car Bruno excelle dans l'art de l'équivoque, ce qui permet au lecteur éventuel de se troubler et de se projeter à son tour dans sa propre lecture. Tel est le cas de Toland qui lit ainsi l'intention du Spaccio en déclarant :

«En vérité, c'est un fait que Scioppius n'a pas saisi partout la pensée de [Brunus], notamment parce qu'il croit que le petit livre De Besta Triumphante vise le Pape; alors qu'il n'y est fait nulle part mention du Pape, et que cette Bête [désigne] toutes les sortes de superstitions qui règnent largement sur les hommes crédules (c'est ce qu'il veut dire), en tous temps et en tous lieux ${ }^{13}$.

11. Ibid., p. 312 : «At ibi de Bruni morte non loquitur Nicodemus, sed de variis sententiis ipsi ab Inquisitoribus ipsi ab Inquisitoribus \& Scioppio malà fide imputatis; ac certo certius est, ista non esse vera omnia, neque verisimilia, quod \& scriptis ejus clarissimè liquet, \& ex ipsa quidem sententiarum repugnantia. Solenne hoc est Inquisitoribus, \& nunquam non usurpatum, illos, quos perdere gestiunt, foede prius denigrare; ut plerisque animi \& corporis vitiis contaminati, nulliusmisericordià, sed omnium potius aversatione, digni censeantur. Quo pacto enimvero Daemonum salutem (exempli gratia) propugnare posset Brunus, ut olim fecerat Origenis, si sacras literas pro somniis duxisset? aut, rejectis scripturis, quinam omnino de Diabolis, vel aeterna salute, sermo ipsi esset instituendus? Viderint haec S. Officii Patres. "

12. Ibid., p. 312-313 : «Sans doute dans ses écrits n'a-t-il jamais entendu par magie autre chose que une sagesse un peu plus ésotérique et inaccessible au vulgaire, bien qu'elle soit tout a fait <maxime> naturelle. C'est ainsi qu'il lui arrive parfois d'appeler Transmigration l'éternelle mutation des formes matérielles; chez lui ce vocable prend souvent ce sens. Il en va du reste comme de ce dont on peut juger ici. »

13. Ibid., p. 313. 
Bref, pour Toland, Bruno est un pourfendeur des superstitions religieuses qui ont perverti la vraie religion, non seulement à l'époque où il vivait, mais de tout temps. C'est à ce titre que l'auteur du Spaccio sert de référence à Toland pour lutter contre les affabulations obscurantistes des religions dites révélées et qui s'autorisent de cette Révélation pour exiger de la part des laïcs une soumission docile aux clercs et au prince.

Sans s'étendre davantage sur cette question, Toland expose les grandes lignes de la cosmologie brunienne en se référant à l'Infinito et à la Causa. Le déiste irlandais voit en Bruno un infinitiste convaincu, car il affirme que l'extension de l'univers est infinie aussi bien que la quantité de matière et la pluralité des mondes qu'il contient. À cet égard, Bruno passe pour un «précurseur» de Descartes et même de Fontenelle ${ }^{14}$. Sur ce point, Toland suit assez fidèlement les enseignements du De l'infinito, dont il avait d'ailleurs traduit en anglais la "Proemiale Epistola " (qui résumait les principaux arguments de l'ouvrage) avec une grande rigueur ${ }^{15}$. Dans le bref aperçu du De genere, loco et tempore mortis Jordani Bruni Nolani, Toland respecte la hiérarchisation brunienne des systèmes de mondes (soleils lumineux par eux-mêmes; planètes et satellites réfléchissant la lumière de leur soleil respectif) qui vient multiplier à l'infini le modèle copernicien du système solaire puisqu'il considère chaque étoile fixe comme un soleil entouré de ses planètes et satellites, c'est-à-dire comme le centre relatif d'un monde organisé. John Toland souscrit lui-même sans réserve à cette cosmologie infinitiste, mais en lui donnant une forte coloration matérialiste dont Bruno s'était pourtant clairement et vigoureusement écarté à plusieurs reprises.

"Cependant, pour ne rien te cacher, il croyait que la totalité des choses était constituée de la seule matière, et même au sens strict qu'elle était une et infinie; et que, pour cette raison, les globes ou les terres, ou plutôt les planètes et les mondes sont innombrables et tournent sans fin dans l'immense extension de l'éther autour de leurs soleils respectifs, autrement dit autour des étoiles fixes. Il affirmait aussi que la plupart d'entre eux [ces mondes], sinon tous, étaient accompagnés de leur cortège de lunes, ou (comme on dit), de satellites ${ }^{16}$.

14. Ibid., p. 314 : « Mais pourtant je ne suis pas homme à accuser ici Fontenelle ou plutôt Descartes de plagiat, même si ce dernier fut l'objet de nombreux soupçons. " Ici, manifestement, Toland fait allusion aux accusations de plagiat que le père Daniel Huet porta contre Descartes en prétendant qu'il s'était plus qu' « inspiré " des ouvrages de Bruno pour sa cosmologie, et notamment du De immenso. Cette accusation de plagiat est reproduite par Bayle dans son Dictionnaire à l'article « Bruno ».

15. Voir in ibid., p. 316-330, la traduction de John Toland intitulee : « An Account of Jordano Bruno's Book Of the infinite Universe and innumerable Worlds : In five Dialogues. »

16. Ibid., p. 313-314. 
Il existe toutefois un point précis de la cosmologie brunienne que rejette totalement John Toland, à savoir l'idée que les mondes que l'univers comprend en lui soient altérables, dissolubles et sujets à la mort. En effet, Bruno, qui semblait encore assez imprécis sur cette question dans ses Dialogues italiens ${ }^{17}$, a pris une position claire et définitive dans son $D e$ immenso et innumerabilibus (1591) en faveur de la dissolubilité des mondes dans l'univers immuable. Il écrit à ce propos :

« tu devras admettre que tous les grands animaux, que sont ces mondes, meurent aussi (comme il est manifeste pour les petits [animaux]), c'est-à-dire changent, diminuent, se dissolvent. La matière, fatiguée de son ancien aspect <species>, est aux aguets, désireuse de nouveauté, puisqu'elle désire devenir toute chose et, en raison de ses propres forces, être semblable à tout être : comme le comprend Anaxagore, et cela est tout à fait possible, si les efflux et les influx passent respectivement d'un même corps à un autre ${ }^{18}$.

John Toland oppose au mobilisme brunien une cosmologie éternaliste. Cet éternalisme va de pair, pour Toland, avec son infinitisme. En effet, il considère que la durée de l'univers est infinie a parte ante comme a parte post. Ainsi, l'univers n'ayant jamais commencé, il n'aura pas de fin, tout comme la matière. Pour critiquer Bruno sur ce point et lui substituer une cosmologie éternaliste, Toland place le mobilisme brunien devant une alternative dont les deux branches sont également irrecevables. En effet, de deux choses l'une : ou bien l'ordre et la stabilité apparents du système du

17. Cf., par ex., dans la Cena de le ceneri, 1584, Troisième Dialogue, Quatrième Proposition de Nundinio, trad. franç. Yves HerSaNt, Combas, L’Éclat, 1988, p. 80, Giordano Bruno semble admettre que les astres soient sujets à la mort, car ils perdent des atomes et peuvent également en acquérir, puisqu'ils sont dans un échange constant de particules insécables avec les autres corps composés que sont les mondes innombrables. Toutefois, si une vertu ou une puissance divine interviennent, il est également possible qu'elle conserve une structure identique aux astres malgré le renouvellement incessant de leurs particules constitutives. De même, dans son De l'infinito, universo e mondi, 1584, Quatrième Dialogue, trad. franç. Bertrand Levergeois, Paris, Berg, 1987, p. 131, G. Bruno déclare : " Les corps mondains sont en fait dissolubles, bien que du fait de leur vertu intrinsèque ou d'un influx extérieur ils puissent perdurer éternellement. [...] Aussi demeurent-ils constants en nombre bien que leur substance corporelle soit, comme la nôtre, renouvelée jour après jour. " Felice Tocco avait déjà remarqué l'évolution de la pensée de Bruno vers l'affirmation du caractère transitoire et périssable des corps célestes, cf. son célèbre ouvrage : Le Opere latine di Giordano Bruno esposte e confrontate con le italiane, Florence, 1889, p. 230, n. 1.

18. G. Bruno, De immenso et innumerabilibus, Francfort, 1591, liv. II, chap. v, in éd. Fiorentino, Imbriani, Tallarigo, Tocco, Vitelli, Opera latine conscripta, 3 t. et 8 vol., Naples/Florence, 1879-1891, repr, en fac-similé, Stuttgart-Bad Cannstatt, Frommann, 1962, vol. I, t. 1, p. 272. On lit de même dans la glose latine de ce chapitre, p. 274 : « Ces animaux [que sont les astres] se corrompent de la même manière que nous nous dissolvons, puisque les mêmes éléments procèdent à la composition d'individus ultérieurs, en se détachant de leur substrat, alors que nous savons au contraire assurément qu'ils sont composés et de là dissolubles, c'est certain, et toute la nature le montre. " C'est nous qui traduisons. 
monde sont l'effet d'un Intellect divin qui garantit la constance de sa structure, ou bien il doit être l'effet du hasard. Or, une Intelligence divine étant par nature immatérielle, elle ne saurait avoir d'action directe sur les masses matérielles, puisqu'il y a hétérogénéité entre la substance matérielle et la substance dite spirituelle. En outre, il ne saurait y avoir d'instance intermédiaire entre la matière et l'esprit puisque les deux ne sauraient rien avoir en commun. Il reste alors à faire appel au hasard des épicuriens, c'est-àdire au jeu fortuit des interactions entre les particules matérielles, mais il ne pourrait à lui seul rendre compte de la totalité de l'ordre complexe ni de la constance ou de la stabilité qui caractérisent la structure de l'univers. Mieux vaut donc réduire à néant le problème du commencement et de la fin des mondes en recourant à un éternalisme délibéré. Cette position recule ainsi à l'infini la question de l'origine de l'ordre cosmique et se contente d'en garantir la constance en faisant appel à la notion un peu vague de lois de la nature. Le matérialisme de Toland est le support de son éternalisme et de son infinitisme cosmologiques. C'est ce que donne à comprendre la fin de la lettre de Toland à son ami Hohendorf :

«Il pensait aussi que ses mondes (je l'avais presque oublié) ne sont pas éternels, en ce qui concerne leur forme présente et leur structure, ce qui est parfaitement absurde : puisqu'il ne peut y avoir aucun intermédiaire entre une certaine Intelligence préminente, qui gouverne et informe toute la matière, et l'existence éternelle ainsi que la disposition de toutes choses, qui en arrivent maintenant à être perçues déjà de cette façon. Il est impossible qu'une mouche, et un monde à plus forte raison, soient l'effet du hasard; ce que je me charge de démontrer contre n'importe quel Épicurien, bien que je sois conscient de mes modestes capacités [intellectuelles]. Et l'on pourrait venir à bout de cette affaire sans plus de difficulté contre les Platoniciens; car j'ai la ferme conviction que ce qui n'a jamais eu de commencement n'aura jamais de fin, de même que tout ce qui a été engendré $\langle$ factum est $>$ sera corrompu <infectum $>$ : donc le monde est ou bien éternel et incorruptible, ou bien il a été créé <creatus $>$ un jour et il périra ${ }^{19}$.

Surtout, l'infidélité de Toland à la pensée de Bruno apparaît manifestement à propos de la question de «l'âme » ou de l'« esprit du monde » que le premier réduisait à une partie de la matière, mais dont la seule différence spécifique avec le reste des corps résidait dans sa grande subtilité et mobilité qui ne la soustrayait pas cependant aux lois de la mécanique. Toland précise explicitement en effet :

« Pour ce qui est de l'âme du monde, il s'exprime d'une manière ambiguë dans son livre italien De infinito, universo, \& mundis [sic]; prends garde de ne pas

19. J. TolAND, op. cit. supra n. 9, p. 314-315. 
confondre [sa pensée] avec celle des Platoniciens: puisqu'il n'entend par esprit <spiritus> rien qui soit distinct du composé matériel, mais seulement une partie de la matière plus subtile et plus mobile, agissant mécaniquement $<s u b$ tiliorem tantum ac mobiliorem materiae partem, mechanice agentem >. C'est ce qui apparaîtra clairement si tu lis attentivement ces Dialogues dans lesquels il expose d'une manière vraiment brillante et érudite sa doctrine de la pluralité des mondes ${ }^{20}$.

Certes, il va de soi que Toland substitue subrepticement sa propre conception de l'âme du monde à celle de Bruno. En effet, Toland se borne non seulement à plaquer sur la doctrine de Bruno la conception épicurienne de l'âme, ce qui est déjà en soi inadmissible, mais en outre il dote l'univers matériel d'une âme, ce qui était totalement exclu aux yeux de l'épicurisme. Or, il nous faut revenir précisément sur ces deux points.

Il est clair que Toland « emprunte » à Lucrèce sa doctrine de l'âme ainsi que sa terminologie, car le disciple latin d'Épicure écrivait : « la nature de l'esprit et de l'âme est matérielle. [...] Cet esprit est tout à fait subtil [...] et d'une mobilité sans égale " 21 .

Toutefois, jamais Lucrèce ni Épicure n'ont parlé d'action mécanique des composants de l'âme. À ce niveau, John Toland forge sa nouvelle conception de l'âme en amalgamant les enseignements de l'atomisme antique avec ceux de la pensée mécaniste du Xvir siècle. Mais Toland savait bien qu'il innovait en dotant l'univers d'une âme matérielle - il faut lui rendre cette justice -, car il ne pouvait ignorer que les épicuriens et même les atomistes en général se sont toujours opposés catégoriquement à l'idée d'âme du monde 22 .

C'est plutôt à l'égard de Bruno que John Toland n'est pas rigoureusement fidèle. En effet, le Nolain n'est peut-être pas si «matérialiste » que le libre penseur irlandais le prétend. Toland déclare pourtant qu'il se fonde sur les assertions qui figurent dans la Causa :

« Mais en ce qui concerne l'unité indivisible des choses, et l'extension infinie <infinita extensione> de l'univers, il faut se référer à l'autre livre italien de

20. Ibid., p. 313-314.

21. LUCRECE, De natura rerum, III, v. 161-162; v. 179; v. 205, Paris, Les Belles Lettres, t. 1, p. 92-93: « Haec eadem ratio naturam animi atque animai corpoream docet esse. [...] Principio esse aio persubtilem [...] animi natura reperta mobilis egregie.» C'est nous qui soulignons.

22. Il n'est que d'ouvrir, par exemple, le très célèbre De placitis philosophorum de pseudoPlutarque, liv. II, 3, trad. Guy Lachenaud, Paris, Les Belles Lettres, 1993, p. 104, pour lire que : « D'une façon générale, on considère que le monde est pourvu d'une âme et régi par la providence. En revanche, Démocrite, Épicure et tous ceux qui admettent l'existence des

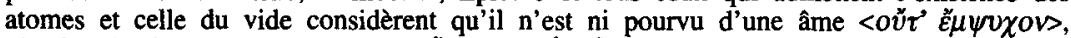

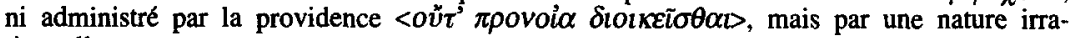
tionnelle. ” 
Brunus, De causa, principio, \& uno; où il soutient que tout ce qui existe est absolument $\langle$ prorsus $\rangle$ matériel ${ }^{23}$.

Ce point est des plus délicats et mérite de notre part une attention toute particulière, car il engage non seulement le sens de la philosophie nolaine, mais aussi la portée véritable du panthéisme de John Toland, comme nous aurons l'occasion de le montrer à propos des Lettres à Serena et du Pantheisticon. Sans chercher des subtilités byzantines à propos de la définition du matérialisme, ce qui n'est pas notre propos ici, il s'agit simplement d'examiner si Bruno a écrit ou laissé entendre que «tout ce qui existe est matériel », comme le prétend son disciple irlandais tardif. Il convient à ce propos de rappeler que tout le dialogue De la causa suit un mouvement d'élévation vers l'Un. Bruno distingue la cause du principe en ce que la première " a son être en dehors de la composition ", tandis que le second " concourt intrinsèquement à la constitution d'une chose et demeure dans l'effet $»^{24}$. Ensuite, il montre que la matière est plutôt un principe qu'une cause et même qu'elle est le principe fondamental de tout ce qui est, donc quelque chose de divin :

«Cette matière, qui explique ce qu'elle tient impliqué, doit être regardée comme divine, comme la meilleure parente, comme la génératrice et la mère des choses naturelles, que dis-je? comme la nature tout entière en substance $"{ }^{25}$.

Contrairement aux enseignements de l'École qui voyait dans la matière une sorte de non-être, Bruno la considère comme un véritable sujet, comme une substance et comme un principe d'être. Toutefois, la conception brunienne de la matière n'est pas des plus simples parce qu'il distingue une matière corporelle et, à l'instar des néo-platoniciens, une matière incorporelle; toutefois, il considère que ces deux types de matières, quelque différents qu'ils soient, ne sont qu'une « seule et même chose ${ }^{26}$. Tout ce que l'on peut dire à ce niveau, c'est que Bruno refuse d'écarter toute substance

23. J. Toland, op. cit. supra n. 9, p. 314 : « De unitate autem rerum indivisibili, \& infinita Universi extensione, videatur alter Bruni libellus Italicus, de causa, principio, \& uno; ubi omne, quod existit, prorsus esse materiale, contendit."

24. G. Bruno, De la causa, principio e uno, 1584, Dialogo secondo, trad. franç. Émile NAMER, Paris, Alcan, 1930, p. 88; éd. ital., G. AquILECCHIA, Turin, Einaudi, 1973, p. 66 : «Causa chami quella che concorre alla produzione delle cose esteriormente. [...] Credo che vogliate che principio sia quello che intrinsecamente concorre alla constituzione della cosa, $e$ rimane nell' effeto." "

25. ID., ibid., trad. franç., p. 183 ; éd. ital., Dialogo quarto, p. 137 : « Quella [materia] dumque che esplica la che tiene implicato, deve essere chiamata cosa divina, et ottima parente, generatrice e madre, di cose naturali : anzi la natura tutta in sustanza.»

26. ID., ibid., trad. franç., p. 173; éd. ital., Dialogo quarto, p. 130 : « anzi l'una e l'altra è una medesima." 
formelle de sa philosophie, et, à cet égard, celle-ci n'est pas un matérialisme strict :

"Démocrite et les Épicuriens prétendent que ce qui n'est pas corps n'est rien, que, par conséquent, la matière seule est la substance des choses, et qu'elle est la nature divine, comme l'a dit un Arabe nommé Avicébron [Ibn Gabirol] dans un livre intitulé Source de vie. Ceux-là ensemble avec les Cyrénaïques, les Cyniques et les Stoïciens veulent que les formes ne soient que d'accidentelles dispositions de la matière. Et moi-même, bien longtemps j'ai adhéré à cette opinion, pour cela seul qu'elle a des fondements qui correspondent plus à la nature que ceux d'Aristote. Mais après mûre réflexion, eu égard à plus d'objets, nous trouvons qu'il est nécessaire de reconnaître dans la nature deux sortes de substances : l'une qui est forme, l'autre qui est matière ${ }^{27}$.

Toutefois, il serait déplacé de voir dans ce passage l'affirmation d'un véritable dualisme métaphysique, c'est-à-dire en l'occurrence d'une sorte d'hylémorphisme traditionnel à peine remanié. Nous savons que le Nolain emprunte souvent des formes d'expression diverses qui n'ont pas manqué de dérouter plus d'un commentateur. En fait, il convient de ne pas perdre de vue que l'objectif essentiel du dialogue italien De la causa c'est une remontée vers l'Un. Ainsi n'est-il pas déplacé de dire que, d'une certaine manière, son naturalisme est une sorte de matérialisme ${ }^{28}$, mais ce vocable est tout simplement très imprécis. On pourrait en dire tout autant du "matérialisme " des stoïciens, que Toland connaissait fort bien. Dans le cas de Bruno, le qualificatif de matérialiste reste beaucoup trop large et trop vague pour caractériser sa pensée de façon significative.

Pour notre part, nous voudrions montrer plutôt ici, qu'avec Giordano Bruno et John Toland, c'est une tout autre façon de penser la nature, l'univers et Dieu qui se met en place dans ce que nous appellerons une logique de l'immanence. Cette pensée immanentiste, John Toland l'appellera, comme on sait, le panthéisme ${ }^{29}$. Ce terme sera bien plus fécond et plus juste pour qualifier les doctrines respectives de Bruno et de Toland, car il met en avant, d'une part, l'idée d'uni-totalité du réel et, d'autre part, celle de Dieu

27. ID., ibid., trad. franç., p. 124 ; éd. ital., Terzo Dialogo, p. 93-94.

28. Comme on sait, telle est l'interprétation générale de BADALONI in La Filosofia di Giordano Bruno, Florence, 1955.

29. À ce sujet, on s'accorde traditionnellement à faire remonter les termes de «panthéisme » et «panthéiste » aux écrits de John Toland, notamment à son Socinianism Truly Stated de 1705. Toutefois, on trouvait déjà le terme de panthéisme sous la plume de Joseph RAPHson, dans son célèbre De spatio reali seu ente infinito conamen mathematico-metaphysicum, Londres, 1697, p. 21, où il écrivait : « Pantheismum etiam hodie apud Indos retinent Brachmanes, qui Deum, seu primam rerum causam per immensam araneam denotant, omnia ex suis texentem visceribus, quae tamen aliquando retrahet forsan, \& in seipsum absorbebit; inter Europaeos insuper Pantheismum profiteri videntur Fanatici quidam \& Enthusiastae, sed horum meminisse, vix operae pretium duximus, utpote ab historia Philosophica alienum. " 
qui n'apparaissaient pas vraiment sous le vocable de «matérialisme ». Tout se passe comme si John Toland avait voulu faire comprendre à son correspondant qu'il risquerait de commettre un grave contresens sur la doctrine de Bruno en voyant dans l' « âme du monde » un principe transcendant. Ce n'est pas tant la non-immatérialité de l'âme que souligne Toland, que son caractère immanent. D'ailleurs, Bruno s'était efforcé de montrer tout au long du deuxième Dialogue de la Causa, en affirmant la différence de sa doctrine par rapport à celle des « Platoniciens ", que l'âme ou l'Intellect du monde n'est pas une cause transcendante, mais plutôt un principe immanent qui façonne la matière de l'intérieur. John Toland faisait très certainement allusion au passage suivant de Bruno que nous citons ici lorsqu'il rédigea sa notice sur la philosophie nolaine pour Hohendorf :

"L'Intellect universel est la faculté intime la plus réelle et la plus propre, la partie la plus efficace de l'âme du monde [...] et il est à la production des choses naturelles ce que notre esprit est à la production ordonnée des espèces rationnelles. Les Pythagoriciens l'appellent le moteur et l'agitateur de l'univers, ainsi que l'a expliqué le poète qui dit :

\section{Totamque infusa per artus \\ Mens agitat molem et toto se corpore miscet.}

Les Platoniciens le nomment forgeron du monde. Ce forgeron, disent-ils, procède du monde supérieur, qui est tout unité, au monde sensible, qui est multiple. [...] Plotin le dit père et générateur, parce qu'il distribue les semences dans le champ de la nature et qu'il est le plus proche dispensateur des formes. Pour nous, il s'appelle l'artiste interne, parce qu'il forme la matière et la figure du dedans, comme du dedans du germe ou de la racine, il fait sortir et développe le tronc, du tronc les premières branches [...]; du dedans il forme, il figure, il innerve, en quelque sorte, les feuilles, les fleurs, les fruits ${ }^{30}$.

Sur ce point de l'immanence, Toland et Bruno s'accordent profondément. En revanche, ils divergent du tout au tout lorsqu'ils se prononcent sur la nature de l'âme du monde, dans la mesure où Toland affirme sa matérialité, tandis que nulle part Bruno n'accepte une telle idée. Pour Bruno, l'âme du monde n'est pas présente dans le monde à la manière des corps matériels dont chacune des parties occupe une portion déterminée de

30. G. Bruno, op. cit. supra n. 24, trad. franç., p. 89-90; éd. ital., p. 67-68: «L'intelletto universale è l'intimita piu reale, e propria facultà e parte potenziale de l'anima del mondo. [...] come il nostro intelletto, alla congrua produzzione di specie razionali. Questo è chiamato da Pitagorici motore et esagitator del universo come esplico il poeta, che disse Totamque infusa per arctus [sic], Mens agitat molem, et toto se corpore miscet. Questo è nomato da Platonici fabro del mondo. [...] Da noi si chiama artefice interno, perché forma la materia, e la figura da dentro, come da dentro del seme o radice manda et esplica il stipe, da dentro il stipe caccia $i$ rami, da dentro i rami le formate brancie, [...] da dentro formaz, figura, intesse, come di nervi, le frondi, gli fiori, gli frutti. " 
l'espace, car « elle n'est pas présente partout [...] d'une façon corporelle et extensive, $[\ldots]$ mais tout entière partout spirituellement $»^{31}$. L'option matérialiste de Toland confère davantage d'homogénéité et de simplicité à sa propre cosmologie qu'à celle de Bruno; en revanche, sa conception mécaniste de l'âme du monde l'écarte considérablement de la conception brunienne du principe formel agissant intrinsèquement dans l'intériorité des êtres.

\section{III. - LE DÉVELOPPEMENT DES ARGUMENTS INFINITISTES DANS LES LETTRES À SERENA}

C'est certainement dans ses Lettres à Serena que John Toland expose le plus clairement sa conception de l'infini, de la matière, de l'espace, du temps et du mouvement des corps. D'ailleurs, l'auteur de ces Lettres doit être d'autant plus persuasif qu'il entend du même coup rectifier, critiquer ou même renverser certains points de la philosophie naturelle de Descartes, Morus, Clarke, Raphson et même des deux grands penseurs qu'il admire tant, à savoir Newton et Locke. Nous envisagerons surtout ici la cinquième Lettre, car c'est elle qui consacre les développements les plus importants à la conception de l'infini.

a) L'abstraction comme source de préjugés sur la nature de l'espace et de l'infini.

Comme nombre de nominalistes illustres, Toland refuse les pièges de l'abstraction, bien qu'il en reconnaisse le caractère indispensable pour des disciplines comme les mathématiques; parmi ces pièges, le pire de tous consiste à réifier des abstractions creuses, puis à prendre ces pseudo-entités comme point de départ pour toutes sortes de constructions intellectuelles dont les conclusions sont finalement frappées de nullité. C'est en effet ce genre de démarche qui aboutit à toutes les erreurs, aux apories et aux difficultés de tous ordres, par suite d'une sorte d'équivoque sur le statut véritable des abstractions intellectuelles. C'est ainsi que John Toland reproche aux mathématiciens d'avoir abusé du recours à l'abstraction et d'avoir laissé croire aux non-spécialistes que leurs définitions nominales pouvaient avoir une valeur réelle. D'où des malentendus regrettables sur la composi-

31. ID., ibid., trad. franç., p. 112; éd. ital., p. 85 : «L'anima del mondo, e la dinività, non sono tutti presenti per tutto e per ogni parte, in modo con cui qualche cosa materiale possa esservi. [...] L'anima del mondo, e forma universale se dicono essere per tutto, non s'intende corporalmente e dimensionalmente [...] ma sono tutti per tutto spiritualmente. » 
tion du continu et de l'espace géométrique à l'aide de points discontinus et inétendus. De même pour le nombre ou quantité discrète susceptible d'un accroissement à l'infini par addition successive de l'unité à elle-même qui laisse croire qu'après un temps infini, on pourrait former un « nombre infini ». Comme on le voit, ces critiques de Toland à l'égard de la pensée mathématique n'est qu'un prélude à la mise en place de sa philosophie naturelle ouvertement infinitiste. Plus précisément, l'infinitisme de Toland n'est qu'une entrée en matière, dans tous les sens du terme : il définit le cadre intellectuel à l'intérieur duquel il pourra expliciter les concepts d'espace ou d'étendue et de matière dont les attributs essentiels sont la solidité et l'activité. John Toland reconnaît lui-même qu'un terme comme celui d'infini a pu donner lieu à tout un ensemble de faux problèmes. Or, si notre free-thinker critique l'abstraction et le pseudo-infini des mathématiciens, c'est-à-dire l'infini potentiel, c'est qu'il entend réhabiliter en même temps l'infini véritable tout en le distinguant radicalement de ce que purent en dire ses contemporains les plus illustres, y compris les infinitistes les plus convaincus.

L'erreur gravissime du potentialisme des mathématiciens, c'est de confondre la propriété d'une opération de l'esprit qui peut être continuellement réitérée (sans se heurter à un quelconque principe interne de limitation) avec une propriété effective des choses, c'est-à-dire une propriété existant réellement en acte. Il ne faut pas confondre un processus avec un être :

"C'est ainsi que le mot infini a donné lieu à de très grands embarras qui ont fait naître une foule d'erreurs \& d'équivoques. On a rendu le nombre infini, comme si de ce que des unités peuvent se joindre à des unités sans fin il s'ensuivoit qu'il existe réellement un nombre infini ; c'est ainsi que l'on a fait un temps infini, on a fait la pensée de l'homme infinie, on a imaginé des lignes asymptotes, \& plusieurs autres progressions sans fin, qui ne sont infinies que relativement aux opérations de notre esprit, sans l'être en elles-mêmes : car ce qui est réellement infini devrait exister actuellement comme tel, au lieu que ce qui n'est que potentiellement infini ne l'est pas positivement ${ }^{32}$.

32. J. Toland, Letters to Serena, Londres, 1704, Cinquième lettre, trad. du baron d'HoLвасн, Paris, 1768, § 11, p. 204; rééd. Stuttgart, Frommann, 1964, p. 179 : « So the word Infinite has bin wonderfully perplex'd; which has given occasion to a thousand Equivocations and Errors. Number was made infinite; as if it follow'd, because Units may be added to one another without end, that there actually existed an infinite Number. Of this nature are infinite Time, the infinite Cogitation of man, asymptot Lines, and a great many other boundless Progressions, which are infinite only with respect to the Operations of our Minds, but not so in themselves, for whatever is really infinite, does actually exist as such; whereas what only may be infinite, is very positively not so. " (Nous abrégeons l'édition française par franç. et la réédition de l'original anglais par angl.) 
L'infini potentiel n'est pas un infini véritable, parce qu'il consiste à lier sériellement du fini à du fini ; c'est pourquoi son infinité n'est que négative, puisqu'elle repose sur l'absence de limitation dans une quelconque opération qui reste cependant toujours inachevée. Laissant ouverte la question de savoir ce qu'est cet infini véritable existant en acte, Toland s'appuie sur sa critique de l'abstraction pour réfuter la conception newtonienne de l'espace et du temps absolus, malgré l'immense admiration qu'il vouait au grand physicien anglais ${ }^{33}$. En effet, c'est au nom de l'absoluité de l'espace (fondée sur le caractère absolu des accélérations, dont Toland n'a pas du tout saisi le sens) que Newton avait affirmé la distinction réelle de l'espace et de la matière. Or, c'est précisément cette distinction qui est inadmissible aux yeux de Toland parce qu'elle conduit directement à l'idée qu'il puisse exister en dehors et indépendamment de la matière un espace incorporel et vide que certains, tel Joseph Raphson, n'ont pas hésité à diviniser. La philosophie naturelle de Toland est une philosophie du plein et c'est la raison pour laquelle ce dernier affirme qu'on pourrait, à la limite, se passer de la notion abstraite d'espace puisque tout ce qui existe est matériel. Du moins, si l'espace existe, ce n'est pas un être substantiel, mais seulement un rapport, une relation entre des êtres qui sont tous matériels :

«L'Espace n'est qu'une notion abstraite, comme vous le verrez par la suite, ou n'est que le rapport qu'un être a avec d'autres êtres qui sont à une distance de lui, sans avoir égard aux choses qui se trouvent entre eux; quoique ces choses aient une existence réelle. Ainsi le Lieu est ou la position relative d'un corps eu égard aux autres corps qui l'environnent, ou la place que ce corps remplit de son propre volume, d'où l'on conçoit que tous les autres corps sont exclus; ce ne sont là que de pures abstractions vu que la capacité ne diffère point du corps contenu. De même la distance est la mesure entre deux corps quelconques sans avoir égard aux choses dont l'étendue est ainsi mesurée. Néanmoins comme les Mathématiciens ont eu besoin de supposer un espace sans matière, de même qu'ils ont supposé une durée sans êtres, des points sans quantité \&c., les Philosophes, qui n'ont point pu sans cela rendre raison de la génération du mouvement dans la matière qu'ils regardoient comme inerte, ont imaginé un espace réel distingué de la matière, qu'ils ont regardé comme étendu, incorporel, immobile, homogène, indivisible, infini ${ }^{34}$.

33. C'est ainsi, par ex., que J. Toland voit dans Newton : «le plus grand des hommes de l'univers ", in op. cit. supra n. 32, Cinquième lettre, $\$ 13$, franç., p. 206, et angl., p. 182 : «I am said to have the greatest Man in the world against me.»

34. Ibid., franç., \& 12, p. 204-205; angl., p. 180-181 : « Space, which is only an abstracted Notion (as you shall perceive hereafter) or the Relation that any thing has to other Beings at a distance from it, without any consideration of what lies between them, tho they have at the same time a real Existence. Thus Place is either the relative Position of a thing with respect to the circumambient Bodys, or the Room it fills with its own Bulk, and from which it is conceiv'd to exclude all other bodys, which are but mere Abstractions, the Capacity nothing fiffering from the Body contain'd: and so Distance is the Measure between any two Bodys, 
La fin de ce passage n'est pas si générale qu'il y paraît. En effet, elle reprend verbatim l'énumération des principaux attributs de l'espace prétendument réel, pour engager une violente polémique contre la philosophie de Joseph Raphson ${ }^{35}$, l'auteur du célèbre De spatio reali seu ente infinito (paru en 1697). Ce dernier avait précisément soutenu, en procédant par voie de synthèse à l'instar des mathématiciens (c'est-à-dire à l'aide de déductions tirées de définitions, axiomes et postulats), que : «l'espace est absolument et par nature indivisible [...], immobile [...], infini [...], acte pur $[\ldots]$, comprenant et pénétrant tout $[\ldots]$, incorporel $[\ldots]$, immuable $[\ldots]{ }^{36}$.

b) L'espace et la matière sont coextensifs et nécessairement infinis.

Toland déploie tous ses efforts pour exposer sa conception de l'espace et de la matière, mais considère qu'il est inutile d'entreprcndre une démonstration particulière pour établir qu'ils ont une existence infinie en acte. En effet, John Toland affirme que l'infinité de l'étendue est devenue désormais une évidence communément reçue ${ }^{37}$. Toutefois, les arguments qu'il cite au passage, ont une forme purement apagogique et reposent globalement sur l'impossibilité d'assigner une quelconque limite absolue à l'espace et à la matière, puisque toute limite est un concept relatif dans la mesure où elle unit ce qu'elle sépare et doit être de même nature que ce qu'elle vient borner :

« Tout le monde convient que l'étendue est infinie vû qu'elle ne peut être bornée par l'inétendue; les démonstrations de ce principe sont si universellement

without reguard to the things whose Extension is so measur'd. Yet because the Mathematicians had occasion to suppose Space without Matter, as they did Duration without Things, Points without Quantity, and the like; the Philosophers, who cou'd not otherwise account for the Generation of Motion in Matter which they held to be inactive, imagin'd a real Space distinct from Matter, which they held to be extended, incorporeal, immoveable, homogeneal, indivisible, and infinite." "

35. Cf. ID., ibid., Cinquième lettre, franç., $\$ 26$, p. 246, où Toland reconnaît d'ailleurs ouvertement qu'il s'en prend nomément à Raphson: "Plusieurs d'entre eux [ceux qui soutiennent l'existence d'un espace absolu, après avoir considéré la matière abstraction faite de l'étendue] ont voulu le [l'espace] faire passer pour l'Être suprême lui-même, ou du moins pour une idée incomplète de la Divinité, comme on peut voir dans le traité de l'espace réel de M. Ralphson [sic] que j'ai eu en vue dans les deux paragraphes précédents; quoique l'on puisse voir d'après les autorités qu'il allègue qu'il n'est point le premier inventeur de cette notion, ni le seul qui la soutienne aujourd'hui <Nay many of them have not stuck to make it pass for the Supreme Being it self, or at least for an inadequate Conception of God, as may be seen in the ingenious Mr. RALPHSON'S Book of Real Space, to whom I had an eye in the two foregoing Paragraphs; tho, as may be likewise learnt from his own Authoritys, he was neither the first Braocher of this conceit, nor the only Maintainer of it now>. 》

36. J. RaPHSON, op. cit. supra n. 29 , chap. v, prop. 1 à 7 , p. 74-76.

37. Toland va même jusqu'à reprocher à Locke, pour qui il a la plus grande admiration, d'avoir repris l'argument traditionnel de la main ou javelot (qui remonte à Epicure et Lucrèce et que Bruno cite fréquemment), pour prouver que l'idée d'un univers clos est impensable. 
reconnues \& adoptées que je ne vous les répète point. La matière n'est pas moins infinie quand on la conçoit comme une substance étendue; car vous ne pouvez point imaginer des limites auxquelles vous ne puissiez ajouter encore de l'étendue à l'infini; ainsi, si elle n'est pas actuellement infinie, sa qualité d'être finie doit venir d'une autre cause que de son étendue ${ }^{38}$.

Certes, il est vrai que cet argument apagogique invoqué ici figure chez de très nombreux auteurs. Mais il fait appel à la continuité de l'espace et surtout à une sorte d'axiome implicite d'homogénéité nécessaire entre le limitant et le limité qui rappelle de façon manifeste l'appareil probatoire du Nolain. C'est ce même argument que Bruno avait développé avec une force toute particulière dans l'Infinito et que Toland connaissait parfaitement puisqu'il en avait traduit le résumé général qui figure dans la lettre liminaire dudit ouvrage :

« Tu auras beaucoup de mal à nous faire entendre comment une chose incorporelle, intelligible et sans dimension puisse être le lieu d'une chose étendue. [...] Parce qu'il est impossible que quelque sens ou quelque imagination, (même si l'on devait retrouver de nouveaux sens et de nouvelles imaginations), puisse sciemment me faire affirmer qu'il se trouve telle surface, telle limite, telle extrémité, au-delà de laquelle il n'y ait ni corps, ni vide, malgré l'existence de Dieu. En effet, la divinité n'a pas à remplir le vide, et par conséquent il ne lui appartient en aucune manière de terminer le corps ${ }^{39}$.

Toutefois, le point faible de l'argumentation de Toland, c'est que l'argument brunien ne peut s'appliquer à la matière qu'à la seule condition d'avoir préalablement démontré que l'existence du vide est impossible. Car on pourrait très bien se figurer l'extension infinie de l'espace qui ne

38. J. Toland, op. cit. supra n. 32, Cinquième lettre, franç., $\S 24$, p. 240 ; angl., p. 213214 : «Extension is granted on all hands to be infinite, for it cannot be terminated by inextension; and the Demonstrations for this are so universally known and acknowledg' d, that I shall not trouble you with repeating them. No less infinite is Matter, when conceiv'd as an extended thing, for you can imagin no bounds of it, to which you may not add more Extension infinitely; and therefore if it be not actually infinite, its Finiteness must proceed from some other Cause besides its Extension. 》

39. Voir De l'infinito, op. cit. supra n. 17 , p. 59 : «tu medesimo sarai molto impacciato per farne intendere come una cosa incorporea, intelligibile e senza dimensione, passa esser luogo di cosa dimensionata. I...J Perche è a fatto impossibile che con qualche senso o fantasia (anco se si ritrovassero altri sensi et altre fantasie) possi farmi affirmare con vera intenzione che si trove tal superficie, tal margine, tal estremità, extra la quale non sia o corpo o vacuo : anco essendovi Dio; perché la divinità non è per impire il vacuo e per conseguenza non è in raggione di quella in modo alcuno di terminare il corpo. " G. BRuNo reprend le même argument dans son De immenso, Francfort, 1591, liv. I, chap. vi, in op. cit. supra n. 18, vol. I, t. 1, p. 222 : « comment peut-il arriver qu'un corps soit limité par quelque chose d'autre qui ne soit pas un corps, que le plan le soit par ce qui n'est pas plan, l'étendue par ce qui n'est pas de l'étendue? <fieri quanam ratione potestur, ullo ut finitum dicas non corpore corpus, non plano planum, spaciosum non spacioso ?> ". C'est nous qui traduisons. 
contiendrait qu'une quantité finie de matière. Telle est d'ailleurs la conception cosmologique développée par Newton et Clarke. C'est pourquoi aussi toute la démonstration de Toland n'a de sens qu'à partir de sa critique initiale de l'abstraction et dont la seule finalité était de montrer que l'espace et la matière sont réellement inséparables, coextensifs et même convertibles : «Pour moi je ne peux pas plus admettre un espace absolu distingué de la matière que le lieu où le placer, ou que je ne puis admettre un temps absolu distingué des choses dont on considère la durée $\gg^{40}$. En outre, cette assertion tirait sa propre force de la conception relationnelle de l'espace que Toland avait judicieusement établie au début de sa démonstration. C'est donc parce que l'espace ne peut être conçu qu'infini et parce qu'il n'est rien d'autre qu'un rapport entre des êtres corporels et étendus, qu'il implique l'existence d'une quantité infinie de matière. Autrement dit, il ne faut jamais oublier que pour Toland, comme pour son ami Leibniz, l'espace n'est pas une substance, il n'est rien par soi ; c'est une entité à structure relationnelle ${ }^{41}$. À l'absolutisme newtonien, Toland oppose la relativité de l'espace et du temps; mais par contrecoup, ce renversement élève la matière au niveau de l'absolu. Ce qui revient à dire qu'il n'y a pas lieu d'espérer atteindre à l'en-soi de l'espace et du temps, puisqu'ils ne sont plus que des dénominations générales et vides de sens dès qu'on les sépare de leur contenu matériel fait de substances étendues et engagées dans la durée. Ou plutôt, l'en-soi des choses, leur être et leur substance, c'est la matière. Or, étant donné que la matière est éternelle et que ses parties doivent nécessairement coexister de façon simultanée, il s'ensuit que son infinité n'a rien de commun avec l'infinité potentielle (c'est-à-dire processive) des mathématiciens : elle ne peut être qu'infinie en acte puisque le temps n'y peut rien changer. De deux choses l'une : la matière est ou bien finie ou bien infinie, mais son extension n'est nullement en devenir. Or, comme la matière ne saurait être limitée par rien, elle est donc infinie en acte.

En utilisant les concepts traditionnels de la métaphysique, Toland avance très brièvement un autre argument en faveur de l'infinité de la matière, mais qui est également apagogique. Celui-ci se borne à montrer que si l'étendue est une propriété, c'est-à-dire la propriété de la chose qui s'étend, elle ne saurait être le fait du néant, mais seulement d'un sujet matériel, car :

40. J. Toland, op. cit. supra n. 32, Cinquième lettre, franç., §13, p. 206-207; angl., p. 182 : " For my part, I can no more believe an absolute Space distinct from Matter, as the place of it; than there is an absolute Time, different from the things whose duration are consider'd."

41. Le relativisme de Toland revient à la définition que ArIstorte en avait donnée dans son traité des Catégories, 7, 6a 36: "On appelle relatives ces choses dont tout l'être consiste en ce qu'elles sont dites dépendre d'autres choses, ou se rapporter de quelque façon à autre chose." 
" comme le néant n'a point de propriétés, l'étendue que tout le monde s'accorde à reconnaître pour infinie, convient à ce sujet qui est infini luimême, \& qui est modifié à l'infini par son mouvement, son étendue et ses attributs inséparables ${ }^{42}$.

Dès lors, d'où vient cette idée de néant qui est, pour ainsi dire, comme le support ontologique de notre concept physique de vide, et partant, de l'idée newtonienne d'espace vide absolu et infini? Toland montre que c'est au dévergondage de l'abstraction qu'il faut attribuer l'origine de nos idées de néant et même de vide. Ces deux idées ne sont rien en elles-mêmes, ce sont des fictions de notre imagination morcelante qui mutile et fragmente le réel en y introduisant des limites, des divisions et des coupures ontologiques qui n'y sont pas :

"Quand on conçoit les corps comme finis, mobiles, divisibles, en repos, pesants ou légers, de différentes formes $\&$ dans des situations variées, alors nous séparons par abstraction les modifications du sujet, ou, si vous voulez, nous séparons les parties du tout, \& nous imaginons des limites propres à certaines portions de la matière qui les séparent $\&$ les distinguent de tout le reste, c'est de là qu'est venue originairement la notion du vide ${ }^{43}$.

De son côté, le «plénisme " de Bruno reposait sur le principe théologique de plénitude, car il faisait valoir, par l'absurde, que : «Ce serait un mal pour cet espace de n'être pas plein ${ }^{44}$. En effet, Dieu étant toutpuissant, ce serait pour lui une forme d'avarice, c'est-à-dire d'imperfection, que de ne créer qu'une quantité finie de corps et de matière alors que sa Sagesse, sa Bonté et sa Puissance sont illimitées. John Toland, pour sa part, n'invoque nullement le principe théologique de plénitude, mais seulement le souci d'éviter la double impasse que constitue soit la réification du vide spatial (impasse newtonienne), soit la divinisation de l'espace (impasse de Joseph Raphson). Toland présente sa démarche qui entend unifier la matière et l'extension comme la seule position philosophique vraiment conséquente :

42. J. Toland, op. cit. supra n. 32, Cinquième lettre, franç., $\$ 24$, p. 242 ; angl., p. 215 : «as Nothing has no Propertys, so that the acknowledg'd infinite Extension belongs to this infinite Subject, which is infinitely modify'd in its Motion, Extension, and other inseparable Attributes. 》

43. Ibid., franç., $\$ 25$, p. 242 ; angl., p. 216 : «When Bodys are conceiv'd finite, movable, divisible, at rest, heavy or light, under different Figures, and in various Situations; then we abstract the Modifications from the Subject, or, if you will, the Parts from the Whole, and imagine proper Boundarys to certain Portions of Matter, which seperate and distinguish them from all the rest, whence came originally the Notion of a Void. 》

44. De immenso, 1591, liv. I, chap. vI, in op. cit. supra n. 18, vol. I, t. 1, p. 223 : « esset ut ergo malum huic spacio non esse repletum. 》 
«Ceux qui soutiennent l'existence d'un espace absolu, après avoir considéré la matière abstraction faite de l'étendue, ont distingué l'étendue générale, de l'étendue particulière de la matière de tel ou tel corps, comme si la dernière était quelque chose de surajouté à la première, quoiqu'ils ne pussent point assigner le sujet de la première étendue, ni dire si c'était une substance qui ne fût ni corps ni esprit ou si c'était une nouvelle espèce de néant, doué pourtant des propriétés de l'être. Bien plus, plusieurs d'entre eux ont voulu le faire passer pour l'Être suprême lui-même ${ }^{45}$.

c) Les trois propriétés de la matière infinie existant en acte : l'extension, l'activité et la solidité.

Outre l'extension infinie de la matière, Toland affirme qu'elle est essentiellement active, en entendant par là qu'elle est continuellement en mouvement, ce qui a l'avantage d'écarter définitivement la question du commencement. Le mouvement a toujours été et sera toujours; on ne peut donc que l'épouser ou le prolonger, mais jamais le commencer ou l'achever ${ }^{46}$. Ce mobilisme universel avait déjà été explicitement affirmé et développé par Giordano Bruno tant dans ses écrits italiens que dans son œuvre latine ${ }^{47}$. Pour Toland, l'activité irrémittente de la matière ne s'oppose nullement, bien au contraire, à la connaissance scientifique des phénomènes physiques. En effet, cette conception dynamiste de la matière doit libérer la recherche scientifique de la nécessité de faire appel à un Deus ex machina pour remettre en ordre sa création, comme c'était encore (malheureusement) le cas de la physique newtonienne. Dans l'univers de Toland, rien ne se perd et rien ne se crée ; c'est de là que proviennent son autarcie et, finalement, son immuabilité sous ses changements :

« Toutes les parties de l'univers sont continuellement dans un mouvement qui produit \& détruit [...]. Il n'est point d'être qui demeure le même pendant une heure de suite; or tous ces changements n'étant que des mouvements de différentes espèces, sont indubitablement des effets d'une action universelle.

45. J. Toland, op. cit. supra n. 32, Cinquième lettre, angl., p. 219 ; franç., § 26, p. 246.

46. Voir ibid., franç., $\$ 12$, p. 181 et angl., p. 188-191, où J. TolaND écrit en effet : « Si la matière elle-même est essentiellement active, on n'a pas besoin de recourir à cette invention pour lui procurer le mouvement, \& il n'est pas nécessaire de chercher la génération de ce mouvement <If Matter it self be essentially active, there's no need to help it to Motion by this Invention, nor is there any Generation of Motion>. "

47. Voir G. BRUNo, De triplici minimo, liv. II, chap. v, in op. cit. supra n. 18, vol. I, t. 3, p. 204 : «Tu ne trouveras pas deux fois la même source, parce que toi aussi tu n'es pas le même, et, de même, on ne peut pas voir deux fois la même flamme d'une torche <veluti nec eundem bis repetes fontem quoque tu non unus et idem, lampadis ut flammam neque cernere quibis eandem>. » C'est précisément le mobilisme universel de Bruno qui le détourna définitivement de tout projet de mathématiser les phénomènes physiques. 
Mais les changements des parties ne produisent aucuns changements dans l'univers; car il est évident que les altérations, les successions, les révolutions, les transmutations continuelles de la matière ne peuvent pas plus accroître ou diminuer la somme de cet univers que l'alphabet ne peut perdre aucune de ses lettres malgré les combinaisons infinies que l'on en fait dans une langue. [...] L'univers ainsi que toutes ses parties demeure toujours le même ${ }^{48}$.

Toutefois, pour établir que l'activité est l'un des trois principaux attributs de la matière, Toland s'est efforcé de combattre le principe d'inertie ${ }^{49}$ qui avait été mis en place avec tant de difficultés par Galilée, Descartes et Newton. De son côté, Newton avait toujours expressément refusé de voir dans la force motrice en général, et surtout dans la force d'attraction, une propriété inhérente à la matière; seule l'inertie pouvait être considérée comme une propriété essentielle et intrinsèque des corps ${ }^{50}$. Sur ce point, Toland semble n'être guère conscient qu'il ne lui est plus possible de se dire véritablement newtonien après avoir écarté la loi d'inertie, l'absoluité de l'espace et du temps, l'existence du vide, la divisibilité de la matière, etc. En outre, étant donné que le concept tolandien d'inertie <inactivity> est trivial et erroné, puisqu'il identifie celui-ci avec une sorte de tendance au repos (tout comme Kepler et Leibniz d'ailleurs), cela ne l'empêche nullement de croire (à tort, cela va de soi) que Newton lui-même s'était aussi opposé à la notion d'inertie sous prétexte qu'il avait intitulé le premier livre de ses Principia: «Des mouvements des corps ${ }^{51}$. Pour Toland, qui fait une critique en règle de la connaissance sensible ${ }^{52}$, tout ce que nous croyons percevoir en repos dissimule, en fait, une activité cachée que seule

48. J. Toland, op. cit. supra n. 32, Cinquième lettre, franç., $\S 15$ et $\S 16$, p. 213-215; angl., p. 188-191 : «All the Parts of the Universe are in this constant Motion of destroying and begetting and destroying. [...] And these Changes being but several kinds of Motion, are therefore the incontestable Effects of some universal Action. But the Changes in the Parts make no Change in the Universe: for it is manifest that the continual Alterations, Successions, Revolutions, and Transmutations of Matter, cause no Accession or Diminution therein, no more than any Letter is added or lost into so many different Words and Languages. [... J The World, with all the Parts and Kinds thereof, continuing at all times in the same condition. "

49. Ibid., franç., $\$ 18-\$ 24$, p. $220-239$; angl, p. 195-212. Cf. spécialement angl., $\$ 21$, p. 202 : "I think after all that has bin said that Action is essential to Matter, since it must be the real Subject of all those Modifications which are call'd local Motions, Changes, Differences, or Diversitys; and principally because absolute Repose, on which the Inactivity or Lumpishness of Matter was built, is entierly destroy'd, and prov'd no where to exist. "

50. Isaac NEwTon avait écrit, en effet, dans ses Principia mathematica philosophiae naturalis, 1687, liv. III, règle III, trad. franç. par $\mathbf{M}^{\mathrm{me}}$ Du CHÂTELET, Paris, 1756-1759, rééd. Paris, Blanchard, 1966, t. II, p. 4 : « Je n'affirme point que la gravité soit essentielle aux corps. Et je n'entends par la force qui réside dans les corps que la seule force d'inertie, laquelle est immuable; au lieu que la gravité diminue lorsqu'on s'éloigne de la Terre. "

51. J. Toland, op. cit. supra n. 32, Cinquième lettre, angl., $\$ 20$, p. 201-202 : « Mr. Newton [...] declares that perhaps no one Body is in absolute Rest, that perhaps no immovable bodily Center is to be found in Nature. [...] and indeed all Physicks ought to be denominated from the Title he has given to the first Book of his Principles, viz. of the Motion of Bodys. "

52. Ibid., angl., § 18, p. 195 sq. 
notre raison peut nous permettre de découvrir ${ }^{53}$. Une fois admis ce dynamisme universel et continuel, sans commencement ni fin ( $a$ parte ante ou $a$ parte post), Toland peut enfin établir que l'Univers matériel jouit d'une sorte d'autosuffisance ${ }^{54}$, le mot n'est pas trop fort, bien que ce statut ontologique ait toujours été réservé à Dieu seul. Quant à la solidité, c'est-à-dire ce que l'on appelait traditionnellement l'impénétrabilité, elle désigne la résistance que les corps opposent aux autres corps lorsqu'ils tentent d'occuper le même lieu en même temps. Ainsi, Toland pense avoir découvert les trois propriétés essentielles de la matière à partir desquelles il devrait être possible de rendre compte désormais de tous les phénomènes. Ces trois propriétés, bien que distinctes les unes des autres, sont étroitement solidaires ${ }^{55}$. On peut même dire que leur indéfectible solidarité est constitutive de l'essence de la matière :

«L'étendue est le sujet immédiat de toutes les divisions, les figures \& les portions de la matière; mais c'est son action qui produit ces changements, \& ils ne pourraient point être distingués sans la solidité. L'action est la cause immédiate de tout mouvement local, de tous les changements $\&$ de toutes les variétés que nous voyons dans la matière; mais l'étendue est le sujet \& la mesure de leurs distances, \& c'est de la solidité que dépend la résistance, l'impulsion \& la protrusion des corps, \& cependant c'est l'action qui les produit dans l'étendue ${ }^{56}$.

Désormais, John Toland peut construire une cosmologie à partir des principes élémentaires et des termes primitifs indéfinissables que sont les trois attributs de la matière infinie. Dans cette perspective, l'extension infinie en acte de l'univers joue un rôle tout à fait éminent dans la stricte mesure où Toland lui-même considère celle-ci comme évidente et communément reçue sans susciter aucune espèce de polémique. Comme son

53. Ibid., angl., $\S 19$, p. 199 : « Since Rest therefore is but a Determination of the Motion of Bodys, a real Action of Resistance between equal Motions, 'tis plain that this is no absolute Inactivity among Bodys, but only a relative Repose with respect to other Bodys that sensibly change their place. "

54. Ibid., franç., $\$ 24$, p. 239 ; angl., p. 213 : « la matière n'étant point inactive, \& n'ayant pas besoin que le mouvement lui soit continuellement imprimé par un agent extérieur <but Matter not being inactive, nor wanting to have Motion continually imprest by an external Agent>. 》

55. Ibid., franç., $\S 29$, p. 259 ; angl., p. 231 : « ce sont trois idées distinctes sans être trois êtres différents, ce sont des façons diverses d'envisager la matière <three distinct Ideas, but not three different things; only the various considerations of one and the self-same Matter. "

56. Ibid., franc., \& 29, p. 258-259; angl., p. 230 : « Extension is the immediate Subject of all the Divisions, Figures, and Parcels of Matter; but t'is Action that causes thoses Alterations, and they cou'd not be distinct without Solidity. Action is the immediate Cause of all local Motions, Changes, or Varietys in Matter; but Extension is the Subject and Measure of their Distances : and tho upon Solidity depends the Resistance, Impulse, and Protrusion of Bodys, yet 'tis Action that produces them in Extension. 》 
illustre prédécesseur infiniste de Nola ${ }^{57}$, Toland pense que si l'on admet d'emblée l'infinité de l'univers, la plupart des faux problèmes posés par la philosophie naturelle disparaîtront d'eux-mêmes, comme par enchantement ${ }^{58}$. Étant donné que les extrémités de l'univers sont fictives, l'infinitisme cosmologique de Toland consacre le triomphe de la raison sur les sens et sur l'imagination morcelante.

\section{IV. - LA STRUCTURE DE L'UNIVERS INFINI AU SENS DU PANTHEISTICON (1720)}

\section{a) L'esquisse d'une cosmologie dans les Lettres à Serena.}

Fort de ses analyses et de ses réfutations en philosophie naturelle, Toland n'a plus qu'à déduire les éléments principaux de sa cosmologie infiniste. Ainsi retient-il huit propriétés principales qui sont solidaires les unes des autres, car l'univers est «immuable, indivisible, infigurable, immense, il englobe tout, il pénètre tout, il est Un, il est le lieu de toutes choses, et il est homogène $" ~{ }^{59}$. Il est tout à fait déconcertant de constater ici que Toland suit presque mot pour mot l'ordre énumératif adopté par Joseph Raphson (auquel il emprunte également ses principaux arguments) et que nous avions déjà rencontré à propos des propriétés de l'espace. Ce qui revient à dire qu'exception faite de la divinisation de l'Espace, Toland rejoint nombre des démonstrations de Raphson ${ }^{60}$, qu'il ne cesse pourtant de dénigrer par ailleurs. Enfin, Toland et Raphson ont pillé l'un comme l'autre littéralement la conception de l'espace et de ses propriétés que Bruno avait développée surtout dans le premier livre de son De immenso ${ }^{61}$.

57. G. Bruno, De l'infinito, op. cit. supra n. 17, p. 65, Premier Dialogue : « Je vois bien à vrai dire qu'envisager le monde, ou l'univers comme vous dites, comme sans terme < (come dite voi l'universo) interminato $>$, ne comporte aucun inconvénient et nous libère d'innombrables soucis auxquels nous enchaîne la thèse inverse. »

58. J. Toland, op. cit. supra n. 32, Cinquième lettre, franç., $\$ 27$, p. 251 ; angl., p. 224 : « sans parler des difficultés insurmontables qui résultent de ces extrémités fictives <not to insist on insurmountable Difficultys arising from those fictitious Extremitys $>$. "

59. Ibid., franç., \$ 25, p. 243-244; angl., p. 216-217 : « the Universe [...] is immovable and indivisible; and also without all Figure, since it has no Bounds or Limits; and immense, since no Quantity, tho never so often repeated, can equal or measure its Extension. [...] all containing. [...] permeates all things; [...] it is one; [...] it is the Place of all things. [...] it is homogeneal. 》

60. De son côté, J. RapHSON qui s'était chargé de faire un historique « complet » des opinions des Anciens et des Modernes sur les attributs de Dieu au chapitre I de son De spatio reali, op. cit. supra n. 29, cite tous les plus grands philosophes, à l'exception de G. Bruno qu'il a pourtant pillé puisqu'il reprend même une démonstration accompagnée d'une figure géométrique complexe qu'il reproduit intégralement sans jamais citer son auteur, voir ibid., chap. Iv, p. 63. Ladite figure se trouve dans le De immenso de G. BRUNo, liv. VII, chap. v, in op. cit. supra n. 18 , vol. I, t. 2, p. 250.

61. G. Bruno, De immenso, liv. I, chap. vmI, in op. cit. supra n. 18, vol. I, t. 1, p. 231-233. 
Au moins, il faut rendre justice à Toland en soulignant qu'il a eu tout de même le courage de reconnaître, contrairement à Raphson, une bonne partie de ses dettes envers Giordano Bruno. Au cas où nous ne l'aurions pas compris, Toland se charge lui-même de nous rappeler, suivant en cela littéralement la formule du Nolain, que « l'Univers est Un, bien qu'il contienne des mondes innombrables <There's but one Universe, tho there may be numberless Worlds $>{ }^{62}$. Or, la cosmologie tolandienne nous réserve encore des surprises, car s'il est vrai qu'elle est fortement imprégnée des démonstrations bruniennes, elle fait appel à la théorie cartésienne des tourbillons pour expliquer l'organisation interne de chacun des systèmes de mondes que compte l'univers :

«Quoique la matière de l'univers soit partout la même, cependant, eu égard à ses différentes modifications, on la conçoit divisée en une infinité de systèmes particuliers \& de tourbillons de matière; ces systèmes de tourbillons se soudivisent encore en d'autres plus ou moins grands, qui dépendent les uns des autres, comme chacun d'eux dépend du tout dans leurs centres, leurs tissus, leurs formes, leur cohérence. Notre Soleil, par exemple, est le centre de l'un de ces grands systèmes qui en renferme un grand nombre d'autres plus petits dans la sphère de son activité, de même que toutes les planètes qui se meuvent autour de lui; ces systèmes sont soudivisés en d'autres plus petits qui en dépendent, comme les satellites de Jupiter dépendent de lui, ou comme la Lune dépend de la Terre ${ }^{63}$.

Cela est d'autant plus surprenant quand on sait que Newton avait explicitement démontré la fausseté et l'impossibilité du modèle tourbillonnaire à partir du théorème des forces centrales ${ }^{64}$. Conformément à sa pratique cou-

62. Ibid.

63. J. Toland, op. cit. supra n. 32, Cinquième lettre, franç., § 15, p. 211-212; angl., p. 187: "Tho the Matter of the Universe be every where the same, yet, according to its various Modifications, it is conceiv'd to be divided into numberless particular Systems, Vortexes, or whirlpools of Matter; and these again are subdivided into other Systems greater or less, which depend on one another, as every one on the Whole, in their Centers, Texture, Frame, and Coherence. Our Sun (for example) is the Center of one of those bigger Systems, which contains a great many lesser ones within the Sphere of its Activity, as all the Planets that move about it: and these are subdivided into yet lesser Systems that depend on them, as his Satellites wait upon Jupiter, and the Moon on the Earth."

64. I. Newton, op. cit. supra n. 50, t. II, scolie de la prop. LII, p. 423-424: « J'ai cherché les propriétés des tourbillons dans cette proposition [prop. LII, p. 416 sq.], afin de connaître s'il était possible d'expliquer les phénomènes célestes pâr des tourbillons. [...] Que si les tourbillons (comme c'est l'opinion de quelques-uns) se meuvent plus vite près du centre, $\&$ ensuite plus lentement jusqu'à un certain éloignement, \& enfin de nouveau plus promptement près de la circonférence; il est certain qu'ils ne pourront observer ni la raison sesquiplée des distances, ni aucune proportion déterminée. C'est donc aux Philosophes à voir comment ils pourront expliquer cette loi de la raison sesquiplée par le moyen des tourbillons. » Voir ibid., 1966, t. I, liv. II, scolie final, p. 426 : « Il est donc certain que les planettes ne sont point transportées par des tourbillons de matière. Car les planettes qui tournent autour du Soleil, selon l'hypothèse de Copernic, font leurs révolutions dans des ellipses qui ont le Soleil dans un de leurs foyers, et elles parcourent des aires proportionnelles au temps. » 
rante, Toland ne retient des auteurs que ce qui semble lui convenir. Cependant, il convient de se tourner vers le Pantheisticon pour trouver la forme la plus achevée de la cosmologie panthéiste de John Toland.

\section{b) L'Univers éternel et infini des panthéistes.}

Lorsqu'on lit le Pantheisticon, on est frappé par le caractère apparemment officiel, institutionnel et quasiment canonique des propos de Toland. Désormais, le ton n'est plus à la discussion ni à la confrontation, comme au temps des Lettres à Serena, mais à l'exposé de la doctrine panthéiste définitivement constituée et opposée radicalement aux chaologistes. D'ailleurs, Toland déclare qu'il ne fait que rapporter sous forme condensée le credo de la « sodalitas socratica » qui répète inlassablement les formules rituelles consacrées.

« Ils assurent que l'Univers (dont le monde que nous connaissons n'est qu' une très petite partie) est infini en étendue comme en puissance; que par la continuité du tout et la contiguité de ses parties il est un; qu'il est immobile dans sa totalité, n'ayant hors de lui ni espace, et mobile à l'égard de ses parties dans des intervalles infinis; incorruptible et nécessaire de l'une et l'autre façon, c'est-à-dire éternel par son existence et par sa durée; qu'il est intelligent par une raison éminente qui a beaucoup de rapport avec notre âme intelligente; enfin que ses parties intégrantes sont toujours les mêmes et que ses parties composantes sont toujours en mouvement. [...] De ce mouvement et de cette intelligence (qui est la force et l'harmonie du Tout infini) naissent des espèces sans nombre, dont chaque individu est en soi-même force et matière; ainsi tout se régit avec une prudence infinie et un ordre parfait dans l'Univers, dans lequel sont compris une infinité de mondes. [...] Enfin, cette force et cette énergie du Tout, qui a tout créé et qui gouverne tout, ayant toujours le meilleur objet pour but, est Dieu, que vous appellerez, si vous voulez, Esprit et Âme de l'Univers, d'où les Associés Socratiques ont été nommés Panthéistes, parce que, selon eux, cette âme ne peut être séparée de l'Univers même, que par le raisonnement ${ }^{65}$.

Ce credo ne fait que rassembler sous une forme dogmatique les thèses qui avaient été établies antérieurement dans les autres écrits du free-

65. J. Toland, Pantheisticon, Londres, 1720 , trad. franç. anonyme du xvine siècle, rééd. par Albert Lantoine, in Un Précurseur de la franc-maçonnerie: John Toland, 1670-1722, Paris, E. Nourry, 1927, \& III et IV, p. 192-193. Cette formule finale de Toland qui affirme que Dieu n'est pas réellement distinct de l'Univers et qui est le noyau dur du panthéisme, n'est qu'un simple plagiat littéral des formules bruniennes. On lit, en effet, sous la plume de G. BruNo in De immenso, liv. VIII, chap. x, in op. cit. supra n. 18, vol. I, t. 2, p. 312 : «Dieu est infini $<$ infinitum> dans l'infini <infinito $>$, partout en toutes choses, il n'est ni au-dessus ni à l'extérieur, mais il est ce qu'il y a de plus intime <praesentissimum $>$ [en toutes choses]; de telle sorte que l'entité <entitas> n'est nullement au-delà ni à l'extérieur des étants <entia>, la nature n'est nullement au-delà des êtres naturels <naturalia>, la bonté n'est nullement au-delà des choses bonnes. Mais on peut distinguer l'essence <essentia> de l'être <ab esse> seulement logiquement <Distinguitur autem essentia ab esse tantum logice>.» 
thinker. Il n'y a donc pas lieu d'y revenir. En revanche, ce sont les considérations cosmologiques et théologiques de Toland que nous analyserons pour finir, dans la mesure où elles apportent quelques éléments nouveaux et significatifs à l'ensemble de la démarche panthéiste.

Tout d'abord, la critique tolandienne de la cosmologie épicurienne se fait plus perspicace que par le passé. En effet, elle avait surtout rejeté auparavant l'idée que les rencontres et assemblages d'éléments puissent être purement fortuits. À présent, Toland voit dans la prétendue chute verticale et éternelle des atomes dans l'espace cosmique infini une inconséquence puisque : « dans un espace infini il ne peut y avoir ni haut ni bas, ni centre ni extrémités $"{ }^{66}$. Ce n'est donc pas à l'atomisme antique que Toland rattache la cosmologie des panthéistes, mais à une tout autre source philosophique, bien plus ancienne cette fois puisqu'elle est censée remonter à l'Égypte ancienne. Toland tombe comme le père Kircher au xviI ${ }^{e}$ siècle et comme Bruno, Patrizi, Ficin et tant d'autres à la Renaissance, dans une sorte d'égyptianisme, c'est-à-dire dans le mythe d'une "prisca philosophia » dont Pythagore et même Copernic seraient des descendants spirituels plus ou moins inspirés. En cela, Toland n'innove pas puisque la plupart des partisans du copernicianisme à la Renaissance et à l'âge classique voyaient dans l'héliocentrisme copernicien un retour de la pensée pythagoricienne :

«Les panthéistes suivent l'astronomie de Pythagore, ou plutôt celle des Égyptiens, et, pour parler selon les modernes, celie de Copernic. Ils placent le Soleil au centre des Planètes, qui font leurs révolutions autour de lui, et entre lesquelles la Terre que nous habitons n'est pas la plus petite ni la plus basse. Ils pensent qu'il y a un nombre infini d'autres Terres semblables à la nôtre qui tournent autour de leurs Soleils (que nous nommons étoiles fixes) dans des temps proportionnés et toujours aux mêmes distances : ils disent la même chose des comètes, qui ont de beaucoup plus grands cercles à décrire. [...] Il n'y a point de véritables irrégularités dans le cours des planètes; il n'y a aucune rétrogradation, aucune station, aucun excentrique, comme cela paraît aux yeux ${ }^{67}$.

On peut constater que ce pythagorisme ou ce copernicianisme, dans la mesure où il multiplie à l'infini les systèmes héliocentriques, n'est autre que celui de Bruno. Toutefois, pour ce qui concerne les forces d'interaction chargées d'assurer la cohésion du Tout, Toland se contente de renvoyer ses lecteurs à l'œuvre scientifique de Newton, ce qui le dispense habilement d'avoir à se prononcer sur les divergences profondes qui séparent sa philosophie naturelle de celle des Principia ${ }^{68}$. La suite de l'exposé sur l'ordre du

66. Ibid., § V, p. 195.

67. lbid., § IX, p. 202.

68. lbid., $\S$ V, p. 195 : « Notre institution nous défend de disputer sur l'action réciproque des globes les uns sur les autres, ou sur les arguments concernant le vide, qui ont été discutés 
monde est tout à fait élémentaire. Mais, curieusement, Toland consacre l'essentiel de ses développements au troisième mouvement de la Terre, le mouvement de précession des équinoxes, pour démontrer que ce dernier découle de la doctrine de la coïncidence des opposés ${ }^{69}$. Tout se passe comme si les philosophes (égyptiens bien entendu ${ }^{70}$ ) avaient déduit $a$ priori le troisième mouvement de la Terre, tandis que les longues observations d'Aristarque, d'Eudoxe, d'Hipparque, de Ptolémée, de Copernic et même de Halley n'auraient fait qu'en constater laborieusement a posteriori le bien-fondé ${ }^{71}$. Après quelques considérations assez confuses ${ }^{72}$ sur les modalités du mouvement de précession des équinoxes, qu'il n'hésite pas à attribuer à toutes les autres planètes de l'univers, Toland en conclut qu'il assure ainsi le retour éternel du même, ce que Bruno avait pourtant refusé expressément ${ }^{73}$.

Après avoir assez rapidement esquissé les rudiments de la cosmologie des panthéistes, Toland aborde dans la seconde partie, qui prend une tournure nettement plus ésotérique, les questions d'ordre théologique. Il passe ainsi du Tout à l' «Un qui est tout entier en toutes choses », c'est-à-dire à Dieu. Cette formule chère au «holenmérisme » de Henry More reprend aussi intégralement les enseignements de l'Infinito et du De la causa de Bruno sans jamais les citer $^{74}$. Comme nombre de ses contemporains,

par plusieurs Philosophes très illustres. Ceux qui voudront apprendre quelque chose de ces matières n'auront qu'à consulter le fameux Newton. "

69. Ibid., § IX-X, p. 203-204: «Que d'agréables énigmes sont ainsi expliquées sans peine par les élèves des Panthéistes! [...] Mais seulement pour dire un mot en passant de leur doctrine Sur la coïncidence des extrêmes (s'il est permis de parler de la sorte). [...] De cette Cö̈ncidence des extrêmes, nos philosophes infêrent que la Terre a un troisième mouvement, véritablement azdmirable, qui se mesure par le mouvement progressif des points équinoxiaux et par la lente mais continuelle déclinaison de la Méridienne. »

70. J. Toland écrit plus bas, in ibid., § XI, p. 205-206: «Ô combien de fois me suis-je moqué de ceux qui méprisaient les Égyptiens sans entendre seulement leurs termes et sans avoir aucune connaissance de la Saine Astronomie, se contentant de faire des cercles inintelligibles et éblouissant l'esprit du petit peuple par des prodiges supposés. ”

71. Ibid., \& IX-XI, p. 204.

72. Non point que Toland n'ait pas compris en quoi consiste le mouvement de précession des équinoxes, car il lui arrive de s'exprimer assez correctement à ce sujet, in ibid., §11. Mais il sombre dans une erreur totale, in ibid., p. 205, lorsqu'il entreprend de se servir du cône de précession pour montrer que : «le point de la Terre qui répond aujourd'hui au pôle Arctique se trouvera sous le pôle antarctique. »

73. G. BRuno, De immenso, liv. III, chap. vII, in op. cit. supra n. 18, Index, p. 477 : « D'où l'on conclut qu'il est tout à fait inepte d'imaginer cette année du monde qui ramène les mêmes effets pratiquement dans la même place et qui [veut] que les astres retournent exactement dans le même arrangement et dans la [même] conjonction. $»$ De son côté, J. ToLAND affirme dans son Pantheisticon, op. cit. supra n. 65, \$ XIV, p. 211-212: « Ce que nous avons déjà enseigné sur la continuelle déclinaison de la Méridienne, et par conséquent sur le changement de l'Áxe de la Terre [...] nous dirons que tous les globes qui sont dans l'espace infini ont le même sort. "

74. Cf., par ex., G. Bruno, op. cit. supra n. 24, 1930, Cinquième Dialogue, p. 199 : « Vous savez ainsi comme toutes les choses sont dans l'univers et l'univers est dans toutes les choses, comme nous sommes en lui et lui en nous; de sorte que tout concourt en une parfaite unité "; 
J. Toland appuie son panthéisme sur la formule célèbre de l'Évangile : "C'est en lui que nous vivons, que nous nous mouvons et que nous existons ${ }^{75}$. Dans cette logique panthéiste, Dieu est en quelque sorte ce par quoi le Tout est Un, tandis que l'Univers infini est bien le Tout de l'Un puisqu'il épuise la totalité de l'être et ne saurait donc être limité par un Autre de quelque ordre que ce soit. L'Un se déploie dans le Tout; le Tout n'est tel que parce qu'il est Un. Et la connaissance de l'uni-totalité infinie, qui délivre l'être de la crainte ou de la menace que suscitent toute limitation et toute négation, s'accompagne nécessairement d'une joie sereine. $\mathrm{La}$ sagesse du décentrement nous apprend à nous défaire de nos vues tronquées qui prenaient la partie pour le Tout, car l'être est au niveau du Tout. Des Stö̈ciens à Bruno, de Bruno à Spinoza, puis à John Toland c'est la même sagesse qui s'affirme en se diversifiant. Comme le dit un jour Toland à un importun qui lui demandait d'où il était : «Le Soleil est mon père, la Terre est ma mère, le Monde est ma patrie et tous les Hommes sont mes parents $\gg^{76}$.

Jean SEIDENGaRT

(mai 1994).

ID., ibid., éd. ital., p. 147 : "Avete dumque come tutte le cose sono ne l'universo e l'universo è in tutte le cose, noi in quello, quello in noi."

75. J. ToLaND, in op. cit. supra n. 65 , II ${ }^{\mathrm{e}}$ partie, p. 224 , reproduit littéralement le texte des Actes des apôtres, 17, 27-28.

76. Ibid., p. 202. 